\title{
10.
}

\section{Démonstration des formules de M. Jacobi, rélatives à la théorie de la rotation d'un corps solide.}

(Par Mr. Somoff, professeur d'analyse à l'université de St. Petersbourg.)

$\mathbf{O}_{n}$ verra peut-être avec quelque intérêt dans ce mémoire la démonstration des belles formules, rélatives à la théorie de la rotation d'un corps solide, que l'illustre auteur des "Fundamenta nova theoriae functionum elliplicarum" a publiées d'abord dans les comptes rendus de l'Académie des sciences de Paris le 30 Juillet 1849 (T. XXIX No. 5) et puis dans le Journal de M. Liouville.

Je rappellerai avant tout les formules fondamentales de la théorie de、 la rotation d'un corps solide autour d'un point fixe, dans le cas où aucune force n'agit sur le corps. Soient $x_{1}, y_{1}, z_{1}$ les coordonnées d'un point quelconque du corps par rapport à trois axes fixes, dont l'origine $O$ est le point fixe, autour duquel s'effectue la rotation, et $x^{\prime}, y^{\prime}, z^{\prime}$ les coordonnées du même point par rapport aux axes principaux d'inertie, passant par $\boldsymbol{O}$. Désignons par $\boldsymbol{A}, \boldsymbol{B}, \boldsymbol{C}$ les moments d'inertie rélatifs à ces seconds axes, et supposons $\boldsymbol{A}>\boldsymbol{B}>\boldsymbol{C}$. Prenons le plan invariable pour le plan des $x_{1}, y_{1}$ et soient: $\theta$ l'angle $z_{1} O z^{\prime}, \psi$ l'angle compris entre l'axe $\boldsymbol{O x}_{1}$ et l'intersection $\boldsymbol{O N}$ des plans $x_{1} \boldsymbol{O}_{y_{1}}$ et $x^{\prime} \boldsymbol{O}_{y^{\prime}}$, enfin $\varphi$ l'angle $N O x^{\prime}$. En posant

$$
\begin{aligned}
& x_{1}=a x^{\prime}+b y^{\prime}+c z^{\prime}, \\
& y_{1}=a^{\prime} x^{\prime}+b^{\prime} y^{\prime}+c^{\prime} z^{\prime}, \\
& z_{1}=a^{\prime \prime} x^{\prime}+b^{\prime \prime} y^{\prime}+c^{\prime \prime} z^{\prime},
\end{aligned}
$$

on aura par les formules données par Euler pour la transformation des coordonnées:

$$
\begin{aligned}
& \begin{cases}a=\sin \varphi \sin \psi \cos \theta+\cos \varphi \cos \psi, & b=\cos \varphi \sin \psi \cos \theta-\sin \varphi \cos \psi, \\
a^{\prime}=\sin \varphi \cos \psi \cos \theta-\cos \varphi \sin \psi, & b^{\prime}=\cos \varphi \cos \psi \cos \theta+\sin \varphi \sin \psi,\end{cases} \\
& c=\sin \theta \sin \psi \text {, } \\
& c^{\prime}=\sin \theta \cos \psi \text {, } \\
& c^{\prime \prime}=\cos \theta \text {. }
\end{aligned}
$$

Crelle's Journal f. d. M. Bd. XLII. Heft 2. 
Soient $p, q, r$ les vitesses de rotation autour des axes $\boldsymbol{O} x^{\prime}, \boldsymbol{O} y^{\prime}, \boldsymbol{O} \mathfrak{z}^{\prime}$, considérées comme composantes de la vitesse angulaire instantanée; $l$ la valeur du moment principal des quantités du mouvement, et $h$ la somme des forces vives. Cela posé, on aura

$$
\begin{aligned}
& \{\boldsymbol{C} \partial \boldsymbol{r}+(\boldsymbol{B}-\boldsymbol{A}) \boldsymbol{p q} \partial \boldsymbol{t}=\mathbf{B}, \boldsymbol{B} \partial \boldsymbol{q}+(\boldsymbol{A}-\boldsymbol{C}) \boldsymbol{r} p \partial t=0, \boldsymbol{A} \partial \boldsymbol{p}+(\boldsymbol{C}-\boldsymbol{B}) \boldsymbol{q} \boldsymbol{r} \partial \boldsymbol{t}=\mathbf{0}, \\
& \boldsymbol{A} p^{2}+B q^{2}+C \boldsymbol{r}^{2}=h, \quad A^{2} p^{2}+B^{2} q^{2}+C^{2} r^{2}=l^{2}, \\
& p^{2}=\frac{l^{2}-B h+(B-C) C r^{2}}{(A-B) A}, \\
& q^{2}=\frac{l^{2}-A h+(A-C) C r^{2}}{(B-A) B},
\end{aligned}
$$

(5.) $a^{\prime \prime}=-\sin \varphi \sin \theta=\frac{A p}{l}, b^{\prime \prime}=-\cos \varphi \sin \theta=\frac{B q}{l}, c^{\prime \prime}=\cos \theta=\frac{C r}{l}$,

$$
\partial t=\frac{ \pm \sqrt{ }(\boldsymbol{A B}) . \boldsymbol{C} \partial \boldsymbol{r}}{\sqrt{\left[l^{2}-B h+(B-C) C r^{2}\right]\left[A h-l^{2}+(C-A) C r^{2}\right]}} .
$$

(Voyez le Traité de Mécanique de M. Poisson.)

La formule (6.) peut être écrite sous la forme

$$
\text { (6. bis) } \quad \partial t=\frac{ \pm \sqrt{ }(\boldsymbol{A B})}{\sqrt{(B-C)(A-C)}} \cdot \frac{\partial r}{\sqrt{ }\left(\boldsymbol{r}^{2}-\boldsymbol{Q}\right)\left(\boldsymbol{P}-\boldsymbol{r}^{2}\right)},
$$

si pour abréger on fait

$$
\boldsymbol{P}=\frac{A h-l^{2}}{(\boldsymbol{A}-\boldsymbol{C}) \boldsymbol{C}}, \quad \boldsymbol{Q}=\frac{\boldsymbol{B} h-l^{2}}{(\boldsymbol{B}-\boldsymbol{C}) \boldsymbol{C}}
$$

Dans le cas considéré par M. Jacobi, on a $\boldsymbol{A} \boldsymbol{h}>\boldsymbol{l}^{2}, \boldsymbol{B} \boldsymbol{h}>\boldsymbol{l}^{2}$; par conséquent $\boldsymbol{P}$ et $\boldsymbol{Q}$ sont positifs et $\boldsymbol{P}>\boldsymbol{Q}$. Pour que $\partial t$ soit réel, on doit avoir $\boldsymbol{r}^{2}>\boldsymbol{Q}, \boldsymbol{r}^{2}<\boldsymbol{P}$; donc on peut poser

$$
\boldsymbol{r}^{2}=\boldsymbol{P} \cos ^{2} \varepsilon+\boldsymbol{Q} \sin ^{2} \varepsilon,
$$

$\varepsilon$ étant réel. Si de plus on fait

$$
\boldsymbol{k}^{\prime}=\sqrt{\frac{\boldsymbol{Q}}{\boldsymbol{P}}}=\sqrt{\frac{(A-\boldsymbol{C})\left(\boldsymbol{B} h-l^{2}\right)}{(\boldsymbol{B}-\boldsymbol{C})\left(A h-l^{2}\right)}},
$$

on aura

$$
\begin{aligned}
& \text { (8.) } \quad k=\sqrt{ }\left(1-k^{\prime 2}\right)=\sqrt{\frac{P}{P} Q}=\sqrt{\frac{(A-B)\left(l^{2}-C h\right)}{(B-C)\left(A h-l^{2}\right)}}, \\
& \boldsymbol{r}^{2}=\boldsymbol{P}\left(1-k^{2} \sin ^{2} \varepsilon\right)=\boldsymbol{P} \Delta^{2}(\varepsilon, k), \\
& \partial t=\frac{ \pm \sqrt{ }(\boldsymbol{A B C})}{\sqrt{(B-C)\left(A h-l^{2}\right)}} \cdot \frac{\partial \varepsilon}{\Delta(\varepsilon, k)} .
\end{aligned}
$$

Posant dans cette formule

$$
\sqrt{\frac{(B-C)\left(A h-l^{2}\right)}{A B C}}=n,
$$


10. Somoff, sur la théorie de la rotation d'un corps solide.

et donnant au radical le signe $t$, pour que le temps croisse avec $\varepsilon$, elle devient

d'où l'on tire

$$
n \partial t=\frac{\partial \varepsilon}{\Delta(\varepsilon, l)}
$$

$$
n t+\tau=\int_{0}^{\varepsilon} \frac{\partial \varepsilon}{\Delta(\varepsilon, k)}
$$

$\tau$ désignant une constante arbitraire.

La valeur $n t+\tau$ se trouve ainsi exprimée par une fonction elliptique de première espèce, et c'est elle que M. Jacobi prend pour argument dans ses formules. Faisant $n t+\tau=u$, on aura

$$
\text { (11.) }\left\{\begin{array}{c}
\varepsilon=\operatorname{am}(\boldsymbol{u}), \quad \boldsymbol{r}^{2}=\boldsymbol{P} A^{2} \operatorname{am}(\boldsymbol{u}, \boldsymbol{k}) \\
\boldsymbol{r}= \pm \sqrt{\frac{\left(A h-l^{2}\right)}{(A-C) C} \cdot \Delta \operatorname{am}(\boldsymbol{u})}= \pm \sqrt{\frac{\left(A h-l^{2}\right)}{(A-C) C} \cdot \frac{\sqrt{ } k^{\prime} \Theta_{1}(u)}{\Theta(u)}}
\end{array}\right.
$$

où $\Theta(u), \Theta_{1}(u)$ sont des fonctions bien connues, qui jouent un si grand rôle dans la théorie des fonctions elliptiques.

Après avoir substitué cette valeur de $r$ dans la formule (3.), on trouvera

$$
p^{2}=\frac{\left(l^{2}-\boldsymbol{B} h\right)+(\boldsymbol{B}-\boldsymbol{C})\left(\frac{A h-l^{2}}{A-C}\right) \Delta^{2} \mathrm{am}(u)}{(A-\boldsymbol{B}) \boldsymbol{A}}=\frac{(\boldsymbol{B}-\boldsymbol{C}) \boldsymbol{C}}{(\boldsymbol{A}-\boldsymbol{B}) \boldsymbol{A}}\left(\boldsymbol{P} \Delta^{2} \mathrm{am}(\boldsymbol{u})-\boldsymbol{Q}\right) .
$$

Mettant ici $1-k^{2} \sin ^{2} a m(u)$ au lieu de $A^{2}$ am $(u)$, ainsi que les valeurs de $\boldsymbol{P}$ et $k^{2}$, trouvées plus haut, on aura, toutes réductions faites:

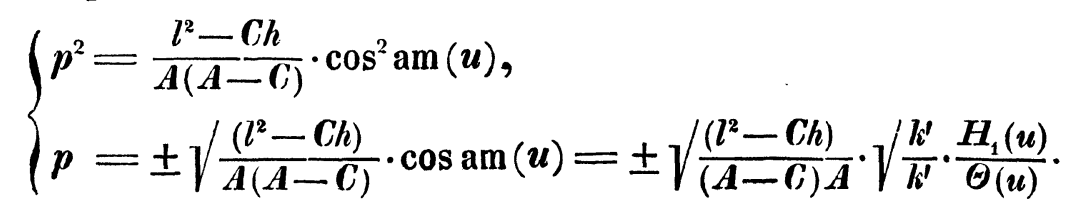

De la mème manière la formule (4.) donne

$$
\left\{\begin{array}{l}
q^{2}=\frac{A h-l^{2}}{(A-B) B} \cdot k^{2} \sin ^{2} \operatorname{am}(u)=\frac{l^{2}-C h}{(B-C) B} \cdot \sin ^{2} \operatorname{am}(u) \\
q= \pm \sqrt{\frac{\left(l^{2}-C h\right)}{(B-C) B}} \cdot \sin \operatorname{am}(u)= \pm \sqrt{\frac{\left(l^{2}-C h\right)}{(B-C) B}} \cdot \frac{H(u)}{\sqrt{ } k \Theta(u)}
\end{array}\right.
$$

Substituant ces valeurs de $p, q, r$ dans les formules (5.) on les réduit par la à

$$
\text { (14.) } \begin{aligned}
a^{\prime \prime}=-\sin \varphi \sin \theta=\frac{A p}{l} & = \pm \frac{1}{l} \sqrt{\frac{A\left(l^{2}-C h\right)}{A-C} \cdot \cos \operatorname{am}(u)} \\
& = \pm \frac{1}{l} \sqrt{\frac{A\left(l^{2}-C h\right)}{A-C} \cdot \sqrt{\frac{l^{\prime}}{k}} \cdot \frac{H_{1}(u)}{\Theta(u)}},
\end{aligned}
$$




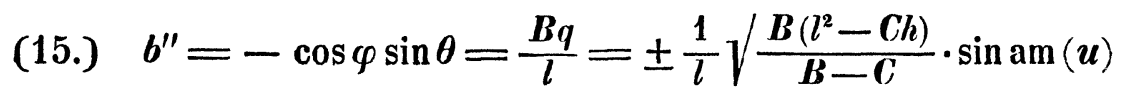

$$
= \pm \frac{1}{l} \sqrt{ } \frac{B\left(l^{2}-C h\right)}{B-C} \cdot \frac{H(u)}{\sqrt{l} \Theta(u)},
$$

(16.) $c^{\prime \prime}=\cos \theta$

$$
\begin{aligned}
& = \pm \frac{1}{l} \sqrt{\frac{C\left(A h-l^{2}\right)}{A-C}} \cdot \Delta \operatorname{am}(u) \\
& = \pm \frac{1}{l} \sqrt{\frac{C\left(A h-l^{2}\right)}{A-C}} \cdot \frac{\sqrt{ } l^{\prime} \Theta_{1}(u)}{\Theta(u)} .
\end{aligned}
$$

Tels sont les cosinus des angles $\left(\boldsymbol{x}^{\prime} \boldsymbol{O} \boldsymbol{z}_{1}\right),\left(\boldsymbol{y}^{\prime} \boldsymbol{O} \boldsymbol{z}_{1}\right),\left(\boldsymbol{z}^{\prime} \boldsymbol{O}_{\mathfrak{z}_{1}}\right)$.

Les formules (14. et 15.) donnent

$$
\operatorname{tg} \varphi= \pm \sqrt{\frac{A(B-C)}{B(A-C)}} \cdot \cot \operatorname{am}(u),
$$

pour calculer l'angle $\varphi$, que fait l'axe $x^{\prime}$ avec la ligne des noeuds $\boldsymbol{O} N$. Le facteur constant $\frac{A(B-C)}{B(A-C)}$ de cette formule est $<1$; donc on pourra poser $\sqrt{\frac{A(B-C)}{B(A-C)}}=\sin \beta$, désignant par $\beta$ une quantité réelle. M. Jacobi considère $\boldsymbol{\beta}$ comme l'amplitude d'un argument elliptique $\boldsymbol{K}^{\prime}-\boldsymbol{a}$, rapporté au module complémentaire $\boldsymbol{k}^{\prime} ; \boldsymbol{K}^{\prime}$ étant l'argument complet rélatif au même module. On a ainsi

$$
\begin{gathered}
\beta=\operatorname{am}\left(\boldsymbol{K}^{\prime}-a, k^{\prime}\right), \quad \boldsymbol{K}^{\prime}-a=\int_{0}^{\beta} \frac{\partial \beta}{\Delta\left(\beta, k^{\prime}\right)}, \quad \boldsymbol{K}^{\prime}=\int_{0}^{\frac{1}{2} \pi} \frac{\partial \beta}{\Delta\left(\beta, k^{\prime}\right)}, \\
a=\boldsymbol{K}^{\prime}-\int_{0}^{\beta} \frac{\partial \beta}{\Delta\left(\beta, k^{\prime}\right)}=\int_{\beta}^{\frac{1}{2} \pi} \frac{\partial \beta}{\Delta\left(\beta, k^{\prime}\right)},
\end{gathered}
$$$$
\sin \beta=\sin \operatorname{coam}\left(a, k^{\prime}\right)=\frac{\cos \mathrm{am}\left(a, k^{\prime}\right)}{\Delta \mathrm{am}\left(a, k^{\prime}\right)}=\sqrt{\frac{A(B-C)}{B(A-C)}} .
$$

De cette dernière formule on tire aisément les expressions suivantes:

$$
\begin{aligned}
& \Delta \cos \operatorname{am}\left(a, k^{\prime}\right)=l \sqrt{\frac{A-B}{B\left(A h-l^{2}\right)}}=\frac{k}{\Delta \operatorname{am}\left(a, k^{\prime}\right)}, \quad \Delta \mathrm{am}\left(a, k^{\prime}\right)=\frac{1}{l} \sqrt{\frac{B\left(l^{2}-C h\right)}{B-C}}, \\
& \cos \operatorname{am}\left(a, k^{\prime}\right)=\frac{1}{l} \sqrt{\frac{A\left(l^{2}-C h\right)}{A-C},} \quad \sin \operatorname{am}\left(a, k^{\prime}\right)=\frac{1}{l} \sqrt{\frac{C\left(A h-l^{2}\right)}{A-C},} \\
& \operatorname{tg} \operatorname{am}\left(a, k^{\prime}\right)=\sqrt{\frac{C\left(A h-l^{2}\right)}{A\left(l^{2}-C h\right)}} .
\end{aligned}
$$

Passant du módule $\boldsymbol{k}^{\prime}$ à $\boldsymbol{k}$ c. à d. de l'argument réel $(a, k)$, à l'argument imaginaire (ai,k), on trouve (Fund. nova \$.19.): 
10. Somoff, sur la théorie de la rotation d'un corps solide.

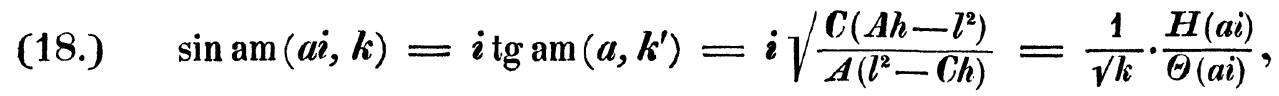

(19.) $\quad \cos \mathrm{am}(a i, k)=\frac{1}{\cos \mathrm{am}\left(a, k^{\prime}\right)} \doteq l \sqrt{\frac{A-C}{A\left(l^{2}-C h\right)}}=\sqrt{\frac{k^{\prime}}{k}} \cdot \frac{H_{1}(a i)}{\Theta(a i)}$,

(20.) $\quad \Delta \mathrm{am}(a i, k)=\frac{\Delta \mathrm{am}\left(a, k^{\prime}\right)}{\cos \mathrm{am}\left(a, k^{\prime}\right)}=\sqrt{\frac{B(A-C)}{A(B-C)}}=\sqrt{ } \boldsymbol{k}^{\prime} \cdot \frac{\Theta_{1}(a i)}{\Theta(a i)}$,

(21.) $\operatorname{tg} \operatorname{am}\left(a i, k^{\prime}\right)=\quad \frac{1}{l} \sqrt{\frac{C\left(A h-l^{2}\right)}{A-C}}=\frac{H(a i)}{\sqrt{l^{\prime} H_{1}(a i)}}$

par conséquent

$$
\text { (22.) }\left\{\begin{array}{l}
\sqrt{\frac{l^{2}-C h}{A(A-C)}}=\frac{l \sqrt{ } k}{A \sqrt{ } k^{\prime}} \cdot \frac{\Theta(a i)}{H_{1}(a i)}=\frac{l}{A} \cdot \frac{1}{\cos a \mathrm{~m}(a i)}, \\
\sqrt{\frac{l^{2}-C h}{B(B-C)}}=\frac{l \sqrt{ } k}{B} \cdot \frac{\Theta_{1}(a i)}{H_{1}(a i)}=\frac{l}{B} \cdot \frac{\Delta a m(a i)}{\cos a m(a i)}, \\
\sqrt{\frac{\left(A h-l^{2}\right)}{C(A-C)}}=\frac{l}{i C \sqrt{ } k^{\prime}} \cdot \frac{H(a i)}{H_{1}(a i)}=\frac{l}{i C} \cdot \operatorname{tg} \operatorname{am}(a i) .
\end{array}\right.
$$

En vertu de ces expressions les formules (11.... 16.) peuvent être remplacées par celles-ci:

(24.)

$$
\begin{aligned}
& \text { (23.) }\left\{\begin{array}{l}
p= \pm \frac{l}{A} \cdot \frac{\Theta(a i) H_{1}(u)}{H_{1}(a i) \Theta(u)}= \pm \frac{l \cos \mathrm{am}(u)}{A \cos \mathrm{am}(a i)} \\
q= \pm \frac{l}{B} \cdot \frac{\Theta_{1}(a i) H(u)}{H_{1}(a i) \Theta(u)}= \pm \frac{l \Delta \mathrm{am}(a i) \sin \mathrm{am}(u)}{B \cos \mathrm{am}(a i)} \\
r= \pm \frac{l}{C} \cdot \frac{H_{(a i)}(u)}{i H_{1}(a i) \Theta(u)}= \pm \frac{l \operatorname{tg} \mathrm{am}(a i) \Delta \mathrm{am}(u)}{i C}
\end{array}\right. \\
& \text { (24.) }\left\{\begin{aligned}
a^{\prime \prime}=-\sin \varphi \sin \theta & = \pm \frac{\Theta(a i) H_{1}(u)}{H_{1}(a i) \Theta(u)}= \pm \frac{\operatorname{cosam}(u)}{\cos \operatorname{am}(a i)} \\
b^{\prime \prime}=-\cos \varphi \sin \theta & = \pm \frac{\Theta_{1}(a i) H(u)}{H_{1}(a i) \Theta(u)}= \pm \frac{\Delta \operatorname{am}(a i) \sin a m(u)}{\cos \operatorname{am}(a i)} \\
c^{\prime \prime}=+\cos \theta & = \pm \frac{H(a i) \Theta_{1}(u)}{i H_{1}(a i) \Theta(u)}= \pm \frac{\operatorname{tgam}(a i) \Delta \operatorname{am}(u)}{i}
\end{aligned}\right.
\end{aligned}
$$

Les signes de $p, q, r$ et ceux des cosinus $a^{\prime \prime}, b^{\prime \prime}, c^{\prime \prime}$ sont toujours les mêmes, et par dépendent par conséquent de la position des axes $x^{\prime}, y^{\prime}, z^{\prime}$ par rapport au plan invariable. Pour bien voir comment se meuvent ces axes dès l'origine de l'argument $u$, nous supposerons $t=0$ avec $u=0$, c. à d. $\boldsymbol{x}=0$; alors on aura pour l'origine du mouvement:

$$
\begin{aligned}
& p= \pm \frac{l}{A \cos a m(a i)}= \pm \sqrt{\frac{l^{2}-C h}{A(A-C)}} \\
& q=0 \\
& r= \pm \frac{l}{i C} \operatorname{tgam}(a i)= \pm \sqrt{\frac{A h-l^{2}}{C(A-C)}}
\end{aligned}
$$


et à cause de $q=0$ on a $b^{\prime \prime}=\frac{A q}{l}=0$ : donc l'axe $O y^{\prime}$, à l'instant $t=0$, est dirigé suivant la ligne des noeuds. Supposons, pour fixer les idées, que, le plan invariable étant horizontal, l'axe du moment $\boldsymbol{O}_{\boldsymbol{z}_{1}}$ soit dirigé dans le sens de la pésanteur, et prenons la direction $\boldsymbol{O} x^{\prime}$ au dessus de ce plan; alors $x^{\prime} \boldsymbol{O}_{\mathfrak{z}_{1}}$ sera $>90^{\circ}$ et par suite $a^{\prime \prime}$ négatif. Il faut donc prendre $p$ à l'origine avec le signe -, savoir $p=-\frac{l}{A \cos a m(a i)} \cdot$ Le temps allant à crôitre, on aura au commencement du mouvement:

$$
p=-\frac{l \cos a m(u)}{A \cos a m(a i)},
$$

et comme $\cos$ am $(u)$ diminue, l'angle $x^{\prime} \boldsymbol{O}_{1}$ diminuera aussi mais restant $>90^{\circ}$. jusqu'à $u=K$, c. à d. jusqu'à $t=\frac{K}{n}$. La direction $\boldsymbol{O} x^{\prime}$ à l'origine est perpendiculaire à la ligne des noeuds; peu après elle forme un angle aigu avec l'une des directions de cette droite, et un angle obtus avec son prolongement. Or un de ces angles est $\varphi$, et comme on suppose $y^{\prime} \boldsymbol{O N}=x^{\prime} \boldsymbol{O N}+90^{\circ}$, on prendra pour $\varphi$ l'angle aigu; donc la direction de $\boldsymbol{O N}$, à l'origine du mouvement, sera opposée à celle de $O y^{\prime}$, et c'est à elle que se rapporte l'angle $\psi$. Le temps $t$ et l'argument $u$ allant à croître, l'angle $y^{\prime} \boldsymbol{O}_{z_{1}}$ deviendra obtus; donc on fera

$$
\cos \left(\boldsymbol{y}^{\prime} \boldsymbol{O}_{\mathbf{z}_{1}}\right)=\boldsymbol{b}^{\prime \prime}=\frac{\boldsymbol{A} \boldsymbol{q}}{l}=-\frac{\Delta \mathrm{am}(\boldsymbol{a} i) \sin \mathrm{am}(u)}{\cos \mathrm{am}(\boldsymbol{a})} ;
$$

par suite $q$ sera négatif. Les valeurs de $p$ et $q$ étant négatives, ainsi que $\boldsymbol{B}-\boldsymbol{A}$, le produit $(\boldsymbol{B}-\boldsymbol{A}) \boldsymbol{p q}$ sera aussi négatif; donc, en vertu de l'équation (1.) on a

$$
\boldsymbol{C} \partial \boldsymbol{r}+(\boldsymbol{B}-\boldsymbol{A}) \boldsymbol{p q} \partial \boldsymbol{t}=\mathbf{0}
$$

où $\partial r$ doit être positif. $\mathrm{Or}$

$$
\partial r=\mp / \frac{A h-l^{2}}{C(A-C)} \cdot k^{2} \sin \operatorname{am}(u) \cos \operatorname{am}(u) \partial u,
$$

et comme $\sin \operatorname{am}(u), \cos a m(u)$ et $\partial u$ sont positifs, il faut prendre

$$
r=-\sqrt{\frac{A h-l^{2}}{C(A-C)}} \cdot \Delta \mathrm{am}(u)=-\frac{l \operatorname{tg} \text { am }(a i)}{i C} \Delta \operatorname{am}(u),
$$

pour que $\partial \boldsymbol{r}$ soit positif. Par suite

$$
\text { (25.) } \quad c^{\prime \prime}=\frac{C^{\prime} r}{l}=\cos \theta=-\frac{\operatorname{tg} \mathrm{am}(a i)}{i} \Delta \mathrm{am}(u)
$$

est aussi négatif; donc la direction de $\boldsymbol{O}_{\boldsymbol{z}}$ doit être prise au dessus du plan invariable. 
10. Somoff, sur la théorie de la rotation d'un corps solide.

Cela posé, on aura

(26.) $\quad \sin \varphi \sin \theta=\frac{\cos \mathrm{am}(u)}{\cos \mathrm{am}(a i)}, \quad \cos \varphi \sin \theta=\frac{\Delta \mathrm{am}(\boldsymbol{a} i) \sin \mathrm{am}(u)}{\cos \mathrm{am}(\boldsymbol{a} i)}$.

La formule (25.) donne

en posant, pour abréger,

$$
\text { (27.) } \quad \sin \theta=\frac{\boldsymbol{R}}{\cos a \mathrm{~m}(a i)},
$$

$$
\boldsymbol{R}=\sqrt{ }\left(1-k^{2} \sin ^{2} \text { am }(\boldsymbol{a i}) \sin ^{2} \mathrm{am}(\boldsymbol{u})\right) .
$$

Ce radical doit être pris avec le signe + , parceque $\sin \theta$ est toujours positif, l'angle $\theta$ ne pouvant excéder $180^{\circ}$. Des formules (26.) on tire

(28.) $\sin \varphi=\frac{\cos \operatorname{am}(u)}{R}, \quad \cos \varphi=\frac{\Delta \operatorname{am}(a i) \sin \operatorname{am}(u)}{R}, \operatorname{tg} \varphi=\frac{\cos \operatorname{am}(u)}{\Delta \operatorname{am}(a i) \sin \operatorname{am}(u)}$.

Cherchons maintenant l'angle $\psi$, compris entre la direction $\boldsymbol{O N}$ et une direction arbitraire $\boldsymbol{O} x^{\prime}$, ménée dans le plan invariable. Pour calculer cet angle, on se servira de l'une des formules:

$$
\text { (29.) } \quad \partial \psi=\frac{h-\boldsymbol{C r}^{2}}{l^{2}-\boldsymbol{C}^{2} \boldsymbol{r}^{2}} l \partial t, \quad \boldsymbol{r} \partial t=\partial \varphi-\cos \theta \partial \psi \text {. }
$$

La première fait voir que $\psi$ diminue quand $t$ et $\boldsymbol{u}$ augmentent. Donc, il faút compter cet angle à partir de $\boldsymbol{O N}$ vers $O \boldsymbol{x}^{\prime}$ dans le sens du mouvement du noeud $N$. La seconde de ces formules est la plus avantageuse pour calculer l'angle $\psi$. On en tire

$$
\text { (30.) } \quad \partial \psi=\frac{\partial \varphi-r \partial t}{\cos \theta} \text {. }
$$

La différentielle de la valeur $\operatorname{tg} \varphi$ (28.) donne

$$
\partial \varphi=-\frac{\cos ^{2} \varphi \partial \operatorname{am}(u)}{\sin ^{2} \operatorname{am}(u) \Delta \operatorname{am}(u)}
$$

et à cause de

$$
\partial \operatorname{am}(u)=\Delta \operatorname{am}(u) \partial u, \quad \cos ^{2} \varphi=\frac{\Delta^{2} \mathrm{am}(a i) \sin ^{2} \mathrm{am}(u)}{R^{2}}
$$

cela se réduit à

$$
\partial \varphi=-\frac{\Delta \mathrm{am}(a i) \Delta \mathrm{am}(u) \partial u}{R^{2}} .
$$

Après avoir substitné dans (30.) cette valeur de $\partial \varphi$, ainsi que celles de $r$ et de $\cos \theta$, trouvées plus haut, on obtient

$$
\partial \psi=\frac{i \Delta \operatorname{am}(a i) \partial n}{R^{2} \operatorname{tg} \operatorname{am}(u i)}-\frac{l \partial u}{C n} .
$$


Pour simplifier cette formule, il faut exprimer $\boldsymbol{n}$ en fonction de l'argument $(\boldsymbol{a} i)$. Or, en vertu des formules (20. et 21.) on a

$$
\sqrt{ }(\boldsymbol{B}-\boldsymbol{C})=\sqrt{\frac{B(A-C)}{A}} \cdot \frac{1}{\Delta \operatorname{am}(a i)}, \quad \sqrt{ }\left(A h-l^{2}\right)=\frac{l}{i} \sqrt{ } \frac{A-C}{C} \cdot \operatorname{tg} \text { am }(a i),
$$

et la substitution dans (10.) donne

$$
\text { (31.) } n=\frac{l(A-C)}{i A C} \cdot \frac{\operatorname{tg} \operatorname{am}(a i)}{\Delta \mathrm{am}(a i)} \text {, }
$$

par suite

$$
\begin{aligned}
\frac{l}{\boldsymbol{C} n} & =\frac{i \boldsymbol{A}}{\boldsymbol{A}-\boldsymbol{C}} \cdot \frac{\Delta \mathrm{am}(a i)}{\operatorname{tg} \mathrm{am}(a i)} \\
\partial \psi & =\frac{i \Delta \mathrm{am}(a i)}{\operatorname{tg} \mathrm{am}(a i)}\left(\frac{1}{\boldsymbol{R}^{2}}-\frac{A}{A-C}\right) \partial u,
\end{aligned}
$$

ou bien aussi, après quelques réductions:

$$
\text { (32.) } \partial \psi=\frac{i k^{2} \sin \mathrm{am}(a i) \cos \mathrm{am}(a i) \Delta \mathrm{am}(a i) \sin ^{2} \mathrm{am}(u) \partial u}{1-k^{2} \sin ^{2} \mathrm{am}(a i) \sin ^{2} \mathrm{am}(u)}-\frac{C}{A-C} \cdot \frac{i \Delta \mathrm{am}(a i)}{\operatorname{tg} \mathrm{am}(a i)} \partial u \text {. }
$$

Il est aisé de voir que $\frac{i \Delta a m(a i)}{\operatorname{tg} a m(a i)}$ est la différentielle de $\log \sin a m(a i)$ par rapport à $a$; car

$$
\frac{\partial \log \sin \operatorname{am}(a i)}{\partial a}=\frac{\cos a m(a i)}{\sin \operatorname{am}(a i)} \cdot \frac{\partial \operatorname{am}(a i)}{\partial a} \text { et } \frac{\partial \operatorname{am}(a i)}{\partial a}=i \Delta \mathrm{am}(a i),
$$

donc

$$
\frac{\partial \log \sin a m(a i)}{\partial a}=\frac{i \Delta \mathrm{am}(a i)}{\operatorname{tg} \operatorname{am}(a i)}
$$

Or $\sin \operatorname{am}(a i)=\frac{H(a i)}{\sqrt{k \Theta(a i)}}$, donc

$$
\text { (33.) } \frac{i \Delta \mathrm{am}(a i)}{\operatorname{tg} \operatorname{am}(a i)}=\frac{\partial \log H(a i)}{\partial u}-\frac{\partial \log \Theta(a i)}{\partial u} \text {. }
$$

Par une propriété fondamentale des fonctions

$$
\boldsymbol{Z}(\boldsymbol{u})=\frac{\partial \log \Theta(u)}{\partial u} \text { (Fund. nova } \$ \text {. 53.) }
$$

on a

(34.) $\frac{k^{2} \sin \mathrm{am}(a i) \cos \mathrm{am}(a i) \Delta \mathrm{am}(a i) \sin ^{2} \mathrm{am}(u)}{1-k^{2} \sin ^{2} \mathrm{am}(a i) \sin ^{2} \mathrm{am}(u)}=Z(a i)+\frac{1}{2} Z(u-a i)-\frac{1}{2} Z(u+a i)$.

En vertu des formules (33. et 34.) l'expression (32.) se réduit à

$$
\begin{aligned}
\partial \psi=-\frac{C}{A-C} \frac{\partial \log H(a i)}{\partial a} \partial u+ & \frac{C}{A-C} \frac{\partial \log \Theta(a i)}{\partial \boldsymbol{a}} \partial u+i Z(\boldsymbol{a} i) \partial \dot{u} \\
& +\frac{1}{2} i[Z(u-a i)-Z(u+a i)] \partial u .
\end{aligned}
$$


Mais

$i Z(\boldsymbol{a} i)=\frac{\partial \log \Theta(a i)}{\partial u}, \quad Z(u-a i)=\frac{\partial \log \Theta(u-a i)}{\partial u}, \quad Z(u+a i)=\frac{\partial \log \Theta(u+a i)}{\partial u}$, donc

$$
\begin{aligned}
\partial \psi=- & \frac{\boldsymbol{C}}{A-\boldsymbol{C}} \frac{\partial \log H(a i)}{\partial \boldsymbol{a}} \partial \boldsymbol{u}+\frac{\boldsymbol{A}}{A-C} \frac{\partial \log \Theta(a i)}{\partial \boldsymbol{a}} \partial \boldsymbol{u} \\
& +\frac{1}{2} i\left[\frac{\partial \log \Theta(u-a i)}{\partial u}-\frac{\partial \log \Theta(u+a i)}{\partial u}\right] \partial u .
\end{aligned}
$$

Posant, pour abréger,

$$
\text { (35.) } \frac{1}{A-C}\left[C \frac{\partial \log H(a i)}{\partial a}-A \frac{\partial \log \Theta(a i)}{\partial a}\right]=n^{\prime},
$$

et prenant l'intégrale, on obtient enfin

$$
\text { (36.) } \quad \psi+n^{\prime} u+D=\frac{1}{2} i \log \left(\frac{\Theta(u-a i)}{\Theta(u+a i)}\right)
$$

$\boldsymbol{D}$ étant une constante arbitraire. Pour la déterminer soit $\psi=\psi_{0}$, pour l'instant $t=0$. On aura

$$
\psi_{0}+\boldsymbol{D}=\frac{1}{2} i \log \left(\frac{\Theta(-a i)}{\Theta(a i)}\right)=\frac{1}{2} i \log \left(\frac{\Theta(a i)}{\Theta(a i)}\right)=0,
$$

donc $\boldsymbol{D}=-\psi_{0}$ et

Posant

$$
\psi-\psi_{0}+n^{\prime} u=\frac{1}{2} i \log \left(\frac{\Theta(u-a i)}{\Theta(u+a i)}\right) .
$$

$$
\text { (37.) } \psi-\psi_{0}+n^{\prime} \boldsymbol{u}=\psi-\psi_{0}+\boldsymbol{n n}^{\prime} t=\Omega
$$

et passant des logarithmes aux nombres, on obtient

$$
\sqrt{ }\left(\frac{\Theta(u+a i)}{\Theta(u-a i)}\right)=e^{i \Omega}, \quad \sqrt{ }\left(\frac{\Theta(u-a i)}{\Theta(u+a i)}\right)=e^{-i \Omega},
$$

et par suite

(38.) $\quad \cos \Omega=\frac{\Theta(u+a i)+\Theta(u-a i)}{2 \sqrt{ }(\Theta(u+a i) \Theta(u-a i))}, \quad \sin \Omega=\frac{\Theta(u+a i)-\Theta(u-a i)}{2 i \sqrt{ }(\Theta(u+a i) \Theta(u-a i))}$.

Par la formule (3.) du \$. 53. des "Fundamenta nova" on a

$$
\begin{gathered}
\sqrt{ }(\Theta(u+a i) \Theta(u-a i)) \\
=\frac{\Theta(u) \Theta(a i)}{\Theta(0)} \sqrt{ }\left(1-k^{2} \sin ^{2} \operatorname{am}(a i) \sin ^{2} \operatorname{am}(u)\right)=\frac{\Theta(u) \Theta(a i)}{\Theta(0)} \boldsymbol{R} ;
\end{gathered}
$$

donc$$
\text { (39.) }\left\{\begin{array}{l}
\cos \Omega=\frac{\Theta(0)[\Theta(u+a i)+\Theta(u-a i)]}{2 \Theta(u) \Theta(a i) R}, \\
\sin \Omega=\frac{\Theta(0)[\Theta(u+a i)-\Theta(u-a i)]}{2 i \Theta(u) \Theta(a i) R},
\end{array}\right.
$$

Crelle's Journal f. d. M. Bd. XLII. Heft 2. 
Il est facile maintenant de déterminer les directions des axes mobiles $O x, O_{y}, O_{z}$, auxquels M. Jacobi rapporte la position du corps. Soit $\boldsymbol{O} \boldsymbol{N}_{0}$

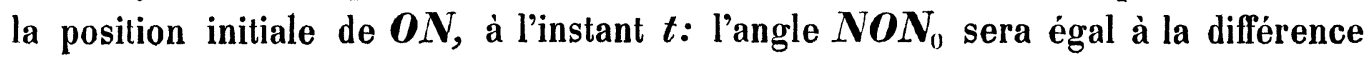
$\psi_{0}-\psi$; donc, si l'on porte sur le plan invariable, a partir de $\boldsymbol{O} \boldsymbol{N}_{0}$ vers $\boldsymbol{O} \boldsymbol{x}_{\boldsymbol{1}}$, l'angle $u n ' t$; le second côté de cet angle déterminera une direction $O x$ de manière que $\angle \boldsymbol{x O N}=\boldsymbol{n} \boldsymbol{n}^{\prime} t-\psi_{0}+\psi=\Omega$. D'ailleurs, il est évident que cette droite aura un mouvement uniforme dans le plan invariable; avec une vitesse $n n^{\prime}$. Cela posé, ménons une perpendiculaire $O_{y}$ à $O x$, de sorte que $\angle y O N_{0}=90^{\circ}-\Omega$. Or les directions $O x, O y$ avec la direction $O_{z}$ opposée à $O_{z_{1}}$, formeront le système mentionné des axes mobiles, auxquels M. Jacobi rapporte la position du corps. Désignant par $x, y, z$ les coordonnées d'un point, rapporté à ces axes, et posant $x=\alpha x^{\prime}+\beta y^{\prime}+\gamma z^{\prime}, y=\alpha^{\prime} x^{\prime}+\beta^{\prime} y^{\prime}+\gamma^{\prime} z^{\prime}, z=\alpha^{\prime \prime} x^{\prime}+\beta^{\prime \prime} y^{\prime}+\gamma^{\prime \prime} z^{\prime}, \theta^{\prime}=180^{0}-\theta$, on trouvera par la transformation des coordonnées, ou bien par la trigonométrie sphérique:

$$
\begin{aligned}
& \alpha=\cos \varphi \cos \Omega-\sin \varphi \sin \Omega \cos \theta^{\prime}, \\
& \alpha^{\prime}=\cos \varphi \sin \Omega+\sin \varphi \cos \Omega \cos \theta^{\prime}, \\
& \alpha^{\prime \prime}=-a^{\prime \prime}=\sin \varphi \sin \theta, \\
& \beta=-\sin \varphi \cos \Omega-\cos \varphi \sin \Omega \cos \theta^{\prime}, \\
& \beta^{\prime}=-\sin \varphi \sin \Omega+\cos \varphi \cos \Omega \cos \theta^{\prime}, \\
& \beta^{\prime \prime}=-b^{\prime \prime}=\cos \varphi \sin \theta, \\
& \gamma=\sin \Omega \sin \theta, \\
& \gamma^{\prime}=-\cos \Omega \sin \theta, \\
& \gamma^{\prime \prime}=\cos \theta^{\prime}=-\cos \theta .
\end{aligned}
$$

Il est maintenant aisé de parvenir aux formules rapportées à la fin de la page $99 \mathrm{du}$ No. cité des "Comples rendus." Il n'y a qu'à substituer dans les formules précédentes les valeurs trouvées de $\sin \varphi, \cos \varphi, \sin \theta, \cos \theta^{\prime}$, $\sin \Omega, \cos \Omega$. (Voyez les formules (25., 26., 27. et 39.)) On aura ainsi $\alpha=\frac{\Theta(0)}{2 \Theta(u) \Theta(a i) \cos \mathrm{am}(a i)}\left[\frac{(\sin \mathrm{am}(u) \cos \mathrm{am}(a i) \Delta \mathrm{am}(a i)+\sin \mathrm{am}(a i) \cos \mathrm{am}(u) \Delta \mathrm{am}(u)) \Theta(u+a i)}{R^{2}}\right]$

$$
+\frac{\Theta(0)}{2 \Theta(u) \Theta(a i) \cos \operatorname{am}(a i)}\left[\frac{(\sin \mathrm{am}(u) \cos \mathrm{am}(a i) \Delta \operatorname{am}(a i)-\sin \operatorname{am}(a i) \cos \operatorname{am}(u) \Delta \operatorname{am}(u)) \Theta(u-a i)}{R^{2}}\right]
$$

Or, par les formules fondamentales de la théorie des fonctions elliptiques, on a 


$$
\begin{gathered}
\frac{\sin \operatorname{am}(u) \cos \operatorname{am}(a i) \Delta \operatorname{am}(a i) \pm \sin \operatorname{am}(a i) \cos \operatorname{am}(u) \Delta \operatorname{am}(u)}{R^{2}} \\
=\sin \operatorname{am}(u \pm a i)=\frac{H(u \pm a i)}{\sqrt{k \cdot \Theta(u \pm a i)}}, \\
\cos \operatorname{am}(a i)=V\left(\frac{k^{\prime}}{k}\right) \frac{H_{1}(a i)}{\Theta(a i)}, \frac{\Theta(0)}{\sqrt{k^{\prime}}}=\Theta(K)=\Theta_{1}(0) .
\end{gathered}
$$

donc, toutes réductions faites, on obtient

$$
\alpha=\frac{\Theta_{1}(0)[H(u+a i)+H(u-a i)]}{2 \Theta(u) H_{1}(u i)} ;
$$

de même on trouvera:

$$
\begin{gathered}
\beta=\frac{-\Theta(0)}{2 \Theta(u) \Theta(a i)}\left[\frac{\cos \mathrm{am}(u+a i) \Theta(u+a i)+\cos \mathrm{am}(u-a i) \Theta(u-a i)}{\cos \operatorname{am}(a i)}\right. \\
=-\frac{\Theta(0)\left[H_{1}(u+a i)+H_{1}(u-a i)\right]}{2 \Theta(u) H_{1}(a i)}, \\
\gamma=\frac{\Theta(0)[\Theta(u+a i)-\Theta(u-a i)]}{2 i \Theta(u) \Theta(a i) R} \cdot \frac{\boldsymbol{R}}{\cos \mathrm{am}(a i)}=\frac{\Theta(0) \sqrt{\frac{k}{k^{\prime}}} \cdot[\Theta(u+a i)-\Theta(u-a i)]}{2 i \Theta(u) H_{1}(a i)},
\end{gathered}
$$

ou, à cause de

$$
\begin{aligned}
& \sqrt{\frac{k}{k^{\prime}}}=\frac{H(K)}{\Theta(0)} \text { (Fund. nova p. 173): } \\
& \gamma=\frac{H_{1}(0)[\Theta(u+a i)-\Theta(u-a i)]}{2 i \Theta(u) H_{1}(a i)} \\
& \alpha^{\prime}=\frac{\Theta(0)}{2 i \Theta(u) \Theta(a i) \cos \operatorname{am}(a i)}[\sin \operatorname{am}(u+a i) \Theta(u+a i)-\sin \operatorname{am}(u-a i) \Theta(u-a i)] \\
& =\frac{\Theta(0)[\Theta(u+a i)-H(u-a i)]}{2 i \Theta(u) \sqrt{ } k^{\prime} \cdot H_{\overline{1}}(a i)}=\frac{\Theta_{1}(0)[H(u+a i)-H(u-a i)]}{2 i \Theta(u) H_{1}(a i)}, \\
& \beta^{\prime}=\frac{\Theta(0)[\cos a m(u-a i) \Theta(u-a i)-\cos a m(u+a i) \Theta(u+a i)]}{2 i \Theta(u) \Theta(a i) \cos \operatorname{am}(a i)}=\frac{\Theta(0)\left[H_{1}(u-a i)-H_{1}(u+a i)\right]}{2 i \Theta(u) H_{1}(a i)}, \\
& \gamma^{\prime}=-\frac{\Theta(0)[\Theta(u+a i)+\Theta(u-a i)] R}{2 \Theta(u) \Theta(a i) R \cos \operatorname{sm}(a i)}=\frac{H_{1}(0)[\Theta(u+a i)+\Theta(u-a i)]}{2 \Theta(u) H_{1}(a i)}, \\
& \alpha^{\prime \prime}=-a^{\prime \prime}=\frac{\Theta(a i) H_{1}(u)}{H_{1}(a i) \Theta(u)}, \beta^{\prime \prime}=-b^{\prime \prime}=\frac{\Theta_{1}(a i) H(a i)}{H_{1}(a i) \Theta(u)}, \gamma^{\prime \prime}=-c^{\prime i}=\frac{H(a i) \Theta_{1}(u)}{i H_{1}(a i) \Theta(u)} .
\end{aligned}
$$

Ces expressions des neuf quantités: $\alpha, \beta, \ldots \gamma^{\prime \prime}$ s'accordent parfaitement avec les formules de M. Jacobi.

La vitesse angulaire de la rotation instantanée $w=\sqrt{ }\left(\boldsymbol{p}^{2}+\boldsymbol{q}^{2}+\boldsymbol{r}^{2}\right)$ peut être décomposée en trois autres $w_{x}, w_{y}, w_{z}$, dont les axes de rotation sont les axes des coordonnées $\boldsymbol{O} x, \mathrm{Oy}_{\boldsymbol{y}} \boldsymbol{O}$. Or pour déterminer ces com- 
posantes, on a

(40.)

$$
\left\{\begin{array}{c}
\boldsymbol{w}_{x}=\alpha \boldsymbol{p}+\boldsymbol{\beta} \boldsymbol{q}+\boldsymbol{\gamma} \boldsymbol{r} \\
=(\boldsymbol{p} \cos \varphi-\boldsymbol{q} \sin \varphi) \cos \Omega-\left[(\boldsymbol{p} \sin \varphi+\boldsymbol{q} \cos \varphi) \cos \theta^{\prime}-\boldsymbol{r} \sin \theta\right] \sin \Omega, \\
\boldsymbol{w}_{y}=\alpha^{\prime} \boldsymbol{p}+\boldsymbol{\beta}^{\prime} \boldsymbol{q}+\boldsymbol{\gamma}^{\prime} \boldsymbol{r} \\
=(\boldsymbol{p} \cos \varphi-\boldsymbol{q} \sin \varphi) \sin \Omega+\left[(\boldsymbol{p} \sin \varphi+\boldsymbol{q} \cos \varphi) \cos \theta^{\prime}-\boldsymbol{r} \sin \theta\right] \cos \Omega, \\
\boldsymbol{w}_{z}=\alpha^{\prime \prime} \boldsymbol{p}+\boldsymbol{\beta}^{\prime \prime} \boldsymbol{q}+\boldsymbol{\gamma}^{\prime \prime} \boldsymbol{r}=-\frac{\boldsymbol{A} \boldsymbol{p}^{2}+\boldsymbol{B} \boldsymbol{q}^{2}+C \boldsymbol{r}^{2}}{l}=-\frac{h}{l}
\end{array}\right.
$$

D'ailleurs, en vertu des formules trouvées plus haut, on a $p \sin \varphi=-\frac{l \cos ^{2} \mathrm{am}(u)}{A R \cos \mathrm{am}(a i)}, \quad q \cos \varphi=-\frac{l \Delta^{2} \mathrm{am}(a i) \sin ^{2} \mathrm{am}(u)}{B R \cos \mathrm{am}(a i)}$, $q \cos \varphi=-\frac{l \Delta \mathrm{am}(a i) \sin \mathrm{am}(u) \cos \mathrm{am}(u)}{A R \cos \mathrm{am}(u i)}, \quad q \sin \varphi=-\frac{l \Delta \mathrm{am}(a i) \sin \mathrm{am}(u) \cos \mathrm{am}(u)}{B R \cos \mathrm{am}(a i)}$ : et par conséquent

(41.) $p \sin \varphi+q \cos \varphi=-\frac{l}{A B} \cdot \frac{B \cos ^{2} a m(u)+A \Delta^{2} a m(a i) \sin ^{2} a m(u)}{R \cos a m(a i)}$,

(42.) $p \cos \varphi-q \sin \varphi=-\frac{l(B-A)}{A B} \cdot \frac{\Delta \operatorname{am}(a i) \sin \operatorname{am}(u) \cos \operatorname{am}(u)}{R \cos a \mathrm{am}(a i)}$.

Après avoir substitué la valeur $1-k^{2} \sin ^{2} a m(a i)$ de $A^{2}$ am(ai), le numérateur de la formule (41.) se présentera sous la forme

$$
\boldsymbol{B}+(\boldsymbol{A}-\boldsymbol{B}) \sin ^{2} \text { am }(\boldsymbol{u})-\boldsymbol{A} \boldsymbol{k}^{2} \sin ^{2} \text { am }(\boldsymbol{a i}) \sin ^{2} \text { am }(\boldsymbol{u}) \text {. }
$$

Or par les formules (18. et 8.) on a

$$
\boldsymbol{A}-\boldsymbol{B}=\frac{-k^{2} A(B-C) \sin ^{2} a m(a i)}{C},
$$

donc la valeur précédente se réduit à

$$
\frac{B}{C}\left[C-A k^{2} \sin ^{2} \operatorname{am}(a i) \sin ^{2} \operatorname{am}(u)\right]
$$

et la formule (41.) se transforme en celle-ci

$$
p \sin \varphi+q \cos \varphi=-\frac{l\left[C-A k^{2} \sin ^{2} a m(a i) \sin ^{2} a m(u)\right]}{A C \cos \operatorname{am}(a i) R}
$$

En vertu de cela et eu égard aux valeurs de $\sin \theta, \cos \theta^{\prime}$ on obtient

$$
\begin{gathered}
(\boldsymbol{p} \sin \varphi+q \cos \varphi) \cos \theta^{\prime}-r \sin \varphi \\
=\frac{r\left[C-A k^{2} \sin ^{2} \mathrm{am}(a i) \sin ^{2} \mathrm{am}(u)\right]}{A \boldsymbol{R} \cos \mathrm{am}(a i)}-\frac{r \boldsymbol{R}}{\cos \mathrm{am}(a i)}=\frac{r(C-A)}{A R \cos \mathrm{am}(a i)}
\end{gathered}
$$

mais par la formule (31.) on a

$$
\frac{C-A}{A}=-\frac{i C n \Delta \mathrm{am}(a i)}{l \lg \operatorname{am}(a i)}
$$


10. Somoff, sur la théorie de la rotation d'un corps solide.

donc

$$
\frac{r(C-A)}{A}=n \Delta \operatorname{am}(a i) \Delta \operatorname{am}(u)
$$

(43.) $\quad(p \sin \varphi+q \cos \varphi) \cos \theta^{\prime}-r \sin \theta=\frac{n \Delta \operatorname{am}(a i) \Delta \operatorname{am}(u)}{R \cos \operatorname{am}(a i)}$.

Tâchons maintenant à simplifier la formule (42.). On tire des formules (8., 19., 20.)

$$
\boldsymbol{B}-\boldsymbol{A}=\frac{-k^{2}(\boldsymbol{B}-\boldsymbol{C})\left(A h-l^{2}\right)}{l^{2}-C h}, \quad \frac{l \Delta \mathrm{am}(a i)}{\boldsymbol{B} \cos a \mathrm{am}(a i)}=V\left(\frac{l^{3}-C^{2} h}{(\boldsymbol{B}-\boldsymbol{C}) \boldsymbol{B}}\right),
$$

done

$$
\frac{l(B-A) \Delta \mathrm{am}(a i)}{A B \cos \operatorname{am}(a i)}-\frac{-k^{2} \sqrt{ }\left((B-C)\left(A h-l^{2}\right)\right) \cdot \sqrt{ }\left(A h-l^{2}\right)}{\left.A \sqrt{ }\left(l^{2}-C h\right) B\right)}
$$

Mlais

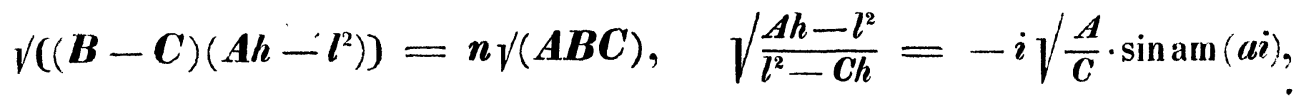

donc

$$
\frac{l(B-A) \Delta \operatorname{am}(a i)}{A B \cos \operatorname{am}(a i)}=i k^{2} n \sin \operatorname{am}(a i)
$$

par conséquent la valeur (42.) se réduit à

(44.) $p \cos \varphi-q \sin \varphi=\frac{-i k^{2} n \sin \operatorname{am}(a i) \sin \operatorname{am}(u) \cos \operatorname{am}(u)}{R}$.

En vertu des formules $(40 ., 43$. et 44.) on obtient

$$
w_{x}=\frac{n \Theta(0)[\Delta \mathrm{am}(u-a i) \Theta(u-a i)-\Delta \mathrm{am}(u+a i) \Theta(u+a i)]}{2 i \Theta(u) \Theta(a i) \cos \operatorname{am}(a i)},
$$

et comme

$$
\Delta \operatorname{am}(u \pm a i)=\sqrt{ } k^{\prime} \cdot \frac{\Theta_{1}(u \pm a i)}{\Theta(u \pm a i)}, \quad \cos \operatorname{am}(a i)=\sqrt{ }\left(\frac{k^{\prime}}{k}\right) \cdot \frac{H_{1}(a i)}{\Theta(a i)},
$$

on a

$$
\text { (45.) } \quad \begin{aligned}
w_{x} & =\frac{n \sqrt{ } k \cdot \Theta(0)\left[\Theta_{1}(u-a i)-\Theta_{1}(u+a i)\right]}{2 i \Theta(u) H_{1}(a i)} \\
& =\frac{n \sqrt{ } k^{\prime} \cdot H_{1}(0)\left[\Theta_{1}(u-a i)-\Theta_{1}(u+a i)\right]}{2 i \Theta(u) H_{1}(a i)}
\end{aligned}
$$

On trouvera de même

$$
\begin{aligned}
w_{y} & =\frac{n \Theta(0)[\Delta \mathrm{am}(u-a i) \Theta(u-a i)+\Delta \mathrm{am}(u+a i) \Theta(u+a i)]}{2 \Theta(u) \Theta(a i) \cos \mathrm{am}(a i)} \\
& =\frac{n \sqrt{ } k^{\prime} \cdot H_{1}(0)\left[\Theta_{1}(u-a i)+\Theta_{1}(u+a i)\right]}{2 \Theta(u) H_{1}(a i)}
\end{aligned}
$$

Enfin

$$
\text { (47.) } w_{z}=-\frac{h}{l} \text {. }
$$


Les formules (45., 46. et 47.) diffèrent des formules de la page 100 du No. cité des Comptes rendus, par les signes de $\Theta_{1}(u+a i), w_{2}$ et par le coefficient $f$, qui est à remplacer, comme on le voit bien, par

$$
n_{\sqrt{ }} \boldsymbol{k}^{\prime}=\sqrt{\frac{(\boldsymbol{B}-\boldsymbol{C})\left(A h-l^{2}\right)}{A B C}} \cdot \sqrt{\frac{(A-C)\left(B \dot{h}-l^{2}\right)}{(B-C)\left(A h-l^{2}\right)}} .
$$

Les formules de la page 100 du No. cité des Comptes rendus, données pour le développement des numérateurs des neuf cosinus $\alpha, \beta, \gamma, \ldots \gamma^{\prime \prime}$ peuvent être déduites très simplement des expressions connues $\Theta(u)=1+2 \sum_{1}^{\infty}(-1)^{m} q^{m^{2}} \cos (2 m x), \quad H(u)=2 \sum_{1}^{\infty}(-1)^{m-1} q^{\frac{z}{(2 m-1)^{2}}} \sin (2 m-1) x$.

Substituant dans ces formules $u \pm a i$ au lieu de $u, x \pm \frac{\pi a i}{2 K}$ au lieu de $x=\frac{\pi u}{2 K}$ et faisant de plus $a=b K^{\prime}$, on aura

$$
\begin{aligned}
& \Theta(u \pm a i)=1+2 \sum_{1}^{\infty}(-1)^{m} q^{m^{2}} \cos \left(2 m x \pm \frac{2 m b \pi K^{\prime} \cdot i}{2 K}\right) \\
& H(u \pm a i)=2 \sum_{1}^{\infty}(-1)^{m-1} q^{4(2 m-1)^{2}} \sin \left[(2 m-1) x \pm \frac{(2 m-1) b \pi K^{\prime} \cdot i}{2 K}\right] .
\end{aligned}
$$

Or

$$
\begin{gathered}
\cos \left(2 m x \pm \frac{2 m b \pi K^{\prime} \cdot i}{2 K}\right)=\frac{1}{2}\left(e^{2 m i x} \cdot e^{\mp \frac{2 m b \pi K^{\prime}}{2 K}}+e^{-2 m i x} \cdot e^{ \pm \frac{2 m b \pi K^{\prime}}{2 K}}\right) \\
=\frac{1}{2}\left(q^{ \pm m b} e^{2 m i x}+q^{\mp m b} e^{-2 m i x}\right),
\end{gathered}
$$

$\sin \left[(2 m-1) x \pm \frac{(2 m-1) b \pi K^{\prime}}{2 K} \cdot i\right]=\frac{q^{ \pm \frac{z}{(2 m-1) b}} \cdot e^{(2 m-1) i x}-q^{\mp z(2 m-1) b} \cdot e^{-(2 m-1) i x}}{2 i}$, donc

$$
\begin{aligned}
& \Theta(u+a i)=1+\sum_{1}^{\infty}(-1)^{m} q^{m^{2}}\left(q^{m b} e^{2 m i x}+q^{-m b} e^{-2 m i x}\right), \cdot \\
& \Theta(u-a i)=1+\sum_{\mathbf{I}}^{\infty}(-1)^{m} q^{m^{2}}\left(q^{-m b} e^{2 m i x}+q^{m b} e^{-2 m i x}\right),
\end{aligned}
$$

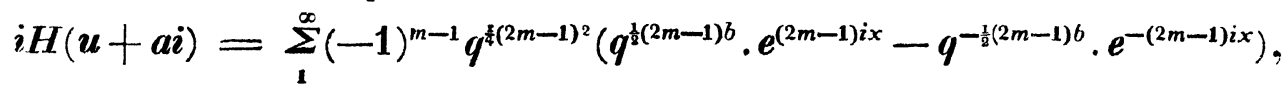

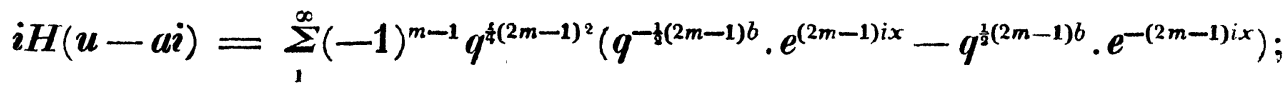

d'où l'on tire

$\frac{1}{2}[\Theta(u+a i)+\Theta(u-a i)]=1+\sum_{1}^{\infty}(-1)^{m} q^{m^{2}-m b}\left(1+q^{2 m b}\right) \cos (2 m x)$,

$\frac{1}{2 i}[\Theta(u+a i)-\Theta(u-a i)]=\sum_{1}^{\infty}(-1)^{m-1} q^{m^{2}-m b}\left(1-q^{2 m b}\right) \sin (2 m x)$,

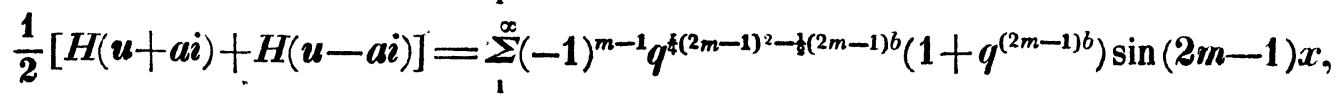

$\frac{1}{2 i}[H(u+a i)-H(u-a i)]=\sum_{1}^{\infty}(-1)^{m-1} q^{4(2 m-1)^{2}-t(2 m-1) b}\left(1-q^{(2 m-1) b}\right) \cos (2 m-1) x$. 
Changeant $u$ en $K-u$ et $x$ en $\frac{1}{2} \pi-x$, on aura $\frac{1}{2}\left[\Theta_{1}(u-a i)+\Theta_{1}(u+a i)\right]=1+\sum^{\infty} q^{m^{2}-m b}\left(1+q^{2 m b}\right) \cos (2 m x)$, $\frac{1}{2 i}\left[\Theta_{1}(u-a i)-\Theta_{1}(u+a i)\right]=\sum_{1}^{\infty} q^{m^{2}-m b}\left(1-q^{2 m b}\right) \sin (2 m x)$, $\frac{1}{2}\left[H_{1}(u-a i)+H_{1}(u+a i)\right]=\sum_{1}^{\infty} q^{\frac{z}{4}(2 m-1)^{2}-\frac{1}{2}(2 m-1) b}\left(1+q^{(2 m-1) b}\right) \cos (2 m-1) x$, $\frac{1}{2 i}\left[H_{1}(u-a i)-H_{1}(u+a i)\right]=\sum_{1}^{\infty} q^{\frac{3}{4}(2 m-1)^{2}-\frac{1}{2}(2 m-1) b}\left(1-q^{(2 m-1) b}\right) \sin (2 m-1) x$.

Ces séries sont très convergentes, parceque $q=e^{-\frac{\pi K^{\prime}}{K}}$ est ordinairement une fraction, dont la valeur ne surpasse pas $\frac{1}{2 \frac{1}{3}}$, et $b<1$. On se bornera aux puissances $q^{9-36}, q^{6-26}$, si l'on ne veut pousser les aproximations que jusqu'au $7^{\text {ine }}$ chiffre décimal.

On parviendra aux formules (1. . . 8.) du No. cité des Comptes rendus en opérant de la manière suivante:

Par les expressions de $\Theta(u), H(u)$ en produits infinis (Fundam. nova \$. 61.) on a

$$
\mathbf{Z}=\frac{\Theta\left(\frac{2 K x}{\pi}+\frac{2 K y}{\pi}\right)}{H\left(\frac{2 K y}{\pi}\right)}
$$

$=\frac{\left[1-2 q \cos 2(x+y)+q^{2}\right]\left[1-2 q^{3} \cos 2(x+y)+q^{6}\right]\left[1-2 q^{5} \cos 2(x+y)+q^{10}\right] \ldots}{2 q^{\frac{2}{4}} \sin y\left[1-2 q^{2} \cos (2 y)+q^{4}\right]\left[1-2 q^{4} \cos (2 y)+q^{6}\right]\left[1-2 q^{6} \cos (2 y)+q^{12}\right] \ldots}$.

Faisant ici $e^{2 i x}=v, e^{2 i y}=z$, on obtient

$$
\begin{aligned}
& 1-2 q^{n} \cos (2 y)+q^{2 n}=\left(1-q^{n} e^{2 i y}\right)\left(1-q^{n} e^{-2 i y}\right)=\left(1-q^{n} z\right)\left(1-q^{n} z^{-1}\right), \\
& 1-2 q^{n} \cos 2(x+y)+q^{2 n}=\left(1-q^{n} v z\right)\left(1-q^{n} v^{-1} z^{-1}\right), \quad \sin y=\frac{z-1}{2 i z^{\frac{1}{2}}}
\end{aligned}
$$

et par suite

$\boldsymbol{Z}=\frac{i z^{\frac{1}{2}}(1-q v z)\left(1-q^{3} v z\right)\left(1-q^{5} v z\right) \ldots \times\left(1-q v^{-1} z^{-1}\right)\left(1-q^{3} v^{-1} z^{-1}\right)\left(1-q^{5} v^{-1} z^{-1}\right) \ldots}{q^{\frac{4}{4}}(z-1)\left(1-q^{2} z\right)\left(1-q^{4} z\right)\left(1-q^{6} z\right) \ldots . \times\left(1-q^{2} z^{-1}\right)\left(1-q^{4} z^{-1}\right)\left(1-q^{6} z^{-1}\right) \ldots}$.

Cette fonction de $z$ peut être décomposée en fractions simples sous la forme

$$
\mathbf{Z}=\frac{i z^{\frac{1}{2}}}{q^{4}}\left[\frac{A_{0}}{z-1}+\sum_{1}^{\infty} \frac{A_{m}}{1-q^{2 m} z}+\sum_{1}^{\infty} \frac{B_{m}}{z-q^{2 m}}\right]
$$

Pour déterminer les coefficients $\boldsymbol{A}_{0}, \boldsymbol{A}_{m}, \boldsymbol{B}_{m}$ on a

$$
\begin{gathered}
\text { (48.) } \quad A_{0}=\left[\frac{q^{4} Z(z-1)}{i z^{\frac{1}{2}}}\right]_{z=1} \\
=\frac{(1-q v)\left(1-q^{3} v\right)\left(1-q^{5} v\right) \ldots \times\left(1-q v^{-1}\right)\left(1-q^{3} v^{-1}\right)\left(1-q^{5} v^{-1}\right) \ldots}{\left(1-q^{2}\right)\left(1-q^{4}\right)\left(1-q^{6}\right) \ldots \times\left(1-q^{2}\right)\left(1-q^{4}\right)\left(1-q^{6}\right) \ldots},
\end{gathered}
$$


110 10. Somoff, sur la théorie de la rotation d'un corps solide.

$$
\begin{gathered}
\boldsymbol{A}_{m}=\left[\frac{q^{\frac{3}{4}} \boldsymbol{Z}\left(1-q^{2 m} z\right)}{i z^{\frac{1}{2}}}\right]_{z=q^{-2 m}} \\
=\frac{\left(1-q^{1-2 m} v\right)\left(1-q^{3-2 m} v\right)\left(1-q^{5-2 m} v\right) \ldots\left(1-q^{-1} v\right)(1-q v) \ldots . \times\left(1-q^{1+2 m} v^{-1}\right) \ldots}{\left(q^{-2 m}-1\right)\left(1-q^{2-2 m}\right)\left(1-q^{4-2 m}\right) \ldots\left(1-q^{-2}\right)\left(1-q^{2}\right) \ldots \times\left(1-q^{2+2 m}\right) \ldots} \\
\boldsymbol{B}_{m}=\left[\frac{q^{\frac{1}{4}} \boldsymbol{Z}\left(z-q^{2 m}\right)}{i z^{\frac{1}{2}}}\right]_{z=q^{2 m}} \\
=\frac{\boldsymbol{q}^{2 m}\left(1-\boldsymbol{q}^{1+2 m} v\right)\left(1-q^{3+2 m} v\right)\left(1-q^{5+2 m} v\right) \ldots \times\left(1-q^{1-2 m} v^{-1}\right)\left(1-q^{3-2 m} v^{-1}\right) \ldots}{\left(\boldsymbol{q}^{2 m}-1\right)\left(1-q^{2+2 m}\right)\left(1-q^{4+2 m}\right) \ldots \times\left(1-q^{2-2 m}\right)\left(1-q^{4-2 m}\right) \ldots}
\end{gathered}
$$

Comparant les expressions de $\boldsymbol{A}_{m}$ et $\boldsymbol{B}_{m}$ à celle de $\boldsymbol{A}_{0}$, il est aisé de voir que

$$
\boldsymbol{A}_{m}=-\boldsymbol{q}^{m} \boldsymbol{v}^{m} \boldsymbol{A}_{0}, \quad \boldsymbol{B}_{m}=\boldsymbol{q}^{m} \boldsymbol{v}^{-m} \boldsymbol{A}_{0}
$$

done

$$
\boldsymbol{Z}=\frac{i z^{\frac{1}{2}} A_{0}}{q^{\frac{4}{4}}}\left[\frac{1}{z-1}-\sum_{1}^{\infty} \frac{q^{m} v^{m}}{1-q^{2 m} z}+\sum_{1}^{\infty} \frac{q^{m} v^{-m} z^{-1}}{1-q^{2 m} z^{-1}}\right]
$$

Posant $\frac{2 K x}{\pi}=u, \frac{2 K y}{\pi}=a i=i b K^{\prime}$, on a $z=e^{-\frac{\pi b K^{\prime}}{K}}=q^{b}, v^{m}=e^{2 m i x}=e^{\frac{\pi u i}{K}}$, $\boldsymbol{Z}=\frac{\Theta(u+a i)}{H(a i)}$, et par conséquent

$$
\frac{\Theta(u+a i)}{H(a i)}=\frac{i A_{0}}{q^{\frac{4}{4}}}\left[\frac{q^{\frac{1}{2} b}}{q^{b}-1}-\sum_{1}^{\infty} \frac{q^{m+\frac{1}{2} b} \cdot e^{2 m i x}}{1-q^{2 m+b}}+\sum_{1}^{\infty} \frac{q^{m-\frac{1}{2} b} \cdot e^{-2 m i x}}{1-q^{2 m-b}}\right] .
$$

Il reste à déterminer la valeur de $\boldsymbol{A}_{0}$. Or d'après l'expression de $\Theta(\boldsymbol{u})$ on a

$$
\frac{\Theta(u)}{\Theta(0)}=\frac{(1-q v)\left(1-q^{3} v\right)\left(1-q^{5} v\right) \ldots \times\left(1-q v^{-1}\right)\left(1-q^{3} v^{-1}\right)\left(1-q^{5} v^{-1}\right) \ldots}{\left[(1-q)\left(1-q^{3}\right) \ldots\right]^{2}}
$$

ce qui réduit la formule (48.) à

$$
\boldsymbol{A}_{0}=\frac{\Theta(u)\left[(1-q)\left(1-q^{3}\right)\left(1-q^{5}\right) \ldots\right]^{2}}{\Theta(0)\left[\left(1-q^{2}\right)\left(1-q^{4}\right)\left(1-q^{6}\right) \ldots\right]^{2}},
$$

el par une formule de la page 89 des "Fuñdam. nova", on a

$$
\frac{\left[(1-q)\left(1-q^{3}\right)\left(1-q^{5}\right) \ldots\right]^{2}}{\left[\left(1-q^{2}\right)\left(1-q^{4}\right)\left(1-q^{6}\right) \ldots\right]^{2}}=\frac{\pi q^{\frac{x}{4}}}{\sqrt{k} \cdot K}
$$

donc

$$
\boldsymbol{A}_{0}=\frac{\Theta(u) \pi q^{\frac{3}{4}}}{\Theta(0) \sqrt{k} \cdot \boldsymbol{K}}
$$

Or, comme

$$
\sqrt{ }\left(\frac{2 K}{\pi}\right)=\Theta(K)=\Theta_{1}(0), \quad \sqrt{ }\left(\frac{2 k K}{\pi}\right)=H(K)=H_{1}(0),
$$

on obtient

$$
\frac{\pi}{\sqrt{k . K}}=\frac{2}{\Theta_{1}(0) H_{1}(0)}
$$


et par suite

$$
\boldsymbol{A}_{0}=\frac{2 \Theta(u) \cdot q^{\frac{x}{4}}}{\Theta(0) \Theta_{1}(0) H_{1}(0)}
$$

Après avoir substitué cette valeur de $\boldsymbol{A}_{0}$ dans la formule (49.), elle se transformera en

$$
\begin{aligned}
\text { (50.) } \quad H_{1}(0) \Theta(0) \Theta_{1}(0) \frac{i \Theta(u+a i)}{2 H(a i) \Theta(u)} \\
=\frac{q^{\frac{1}{2} b}}{1-q^{b}}+\sum_{1}^{\infty} \frac{q^{m+\frac{1}{2} b} \cdot e^{2 m i x}}{1-q^{2 m+b}}-\sum_{1}^{\infty} \frac{q^{m-\frac{1}{2} b} \cdot e^{-2 m i x}}{1-q^{2 m-b}} .
\end{aligned}
$$

Mettant ici $-\boldsymbol{u}$ au lieu de $u$, et remarquant que $\Theta(-\boldsymbol{u}+\boldsymbol{a} i)=\Theta(\boldsymbol{u}-\boldsymbol{a} i)$, $\Theta(-u)=\Theta(u)$ on verra que cette formule se réduit à

$$
\begin{aligned}
& \text { (51.) } \quad H_{1}(0) \Theta(0) \Theta_{1}(0) \frac{i \Theta(u-a i)}{2 H(a i) \Theta(u)} \\
& =\frac{q^{\frac{1}{2} b}}{1-q^{b}}+\sum_{1}^{\infty} \frac{q^{m+\frac{1}{2} b} \cdot e^{-2 m i x}}{1-q^{2 m+b}}-\sum_{1}^{\infty} \frac{q^{m-\frac{1}{2} b} \cdot c^{2 m i x}}{1-q^{2 m-b}} .
\end{aligned}
$$

Prenant la somme et la différence des valeurs (50. 51.) on obtient

$$
\begin{gathered}
H_{1}(0) \Theta(0) \Theta_{1}(0) \cdot \frac{i[\Theta(u+a i)+\Theta(u-a i)]}{2 H(a i) \Theta(u)} \\
=\frac{2 q^{\frac{1}{b} b}}{1-q^{b}}+2\left(q^{\frac{1}{2} b}-q^{-\frac{1}{2} b}\right) \sum_{1}^{\infty} \frac{q^{m}\left(1+q^{2 m}\right) \cos (2 m x)}{\left(1-q^{2 m+b}\right)\left(1-q^{2 m-b}\right)},
\end{gathered}
$$

$H_{1}(0) \Theta(0) \Theta_{1}(0) \cdot \frac{\Theta(u+a i)-\Theta(u-a i)}{2 H(a i) \Theta(u)}=2\left(q^{\frac{1}{3} b}+q^{-\frac{1}{b} b}\right) \sum_{1}^{\infty} \frac{q^{m}\left(1-q^{2 m}\right) \sin (2 m x)}{\left(1-q^{2 m+b}\right)\left(1-q^{2 m-b}\right)}$.

Telles sont précisément les formules (1. et 2. page 101) du No. cité des "Comptes rendus." Les formules (3. et 4.) de la page suivante peuvent aussi être tirées des formules (50.51.). En effet: les formules rapportées au commencement de la page 101, et qui ne diffèrent pas, au fond, des formules du \$. 62 des "Fundam. nova", donnent

$$
\left.\frac{H(u+a i)}{\Theta(a i)}=-e^{-i x} \cdot \frac{\Theta\left[u-i\left(K^{\prime}-a\right)\right]}{H\left[i\left(K^{\prime}-a\right)\right]}\right] \text {. }
$$

En vertu de cette formule, et de (51.), dans laquelle on changera $a$ en $K^{\prime}-a$, et par suite $b$ en $1-b$, on trouve

$$
\begin{gathered}
\text { (52.) } H_{1}(0) \Theta(0) \Theta_{1}(0) \cdot \frac{H(u+a i)}{2 \Theta(a i) \Theta(u)} \\
=-\frac{1}{i}\left[\frac{q^{\frac{1}{2}-\frac{1}{2} b} \cdot e^{-i x}}{1-q^{1-b}}+\sum_{1}^{\infty} \frac{q^{m+\frac{1}{2}-\frac{1}{2} b} \cdot e^{-(2 m+1) i x}}{1-q^{2 m+1-b}}-\sum_{2}^{\infty} \frac{q^{m-\frac{1}{2}+\frac{1}{2} b} \cdot e^{(2 m-1) i x}}{1-q^{2 m-1+b}}\right] .
\end{gathered}
$$

Substituant $-u$ à $u$, et remarquant que $H(-u+a i)=-H(u-a i)$, cette Crelle's Journal f. d. M. Bd. XLII. Heft 2. 
10. Somoff, sur la théorie de la rotation d'un corps solide.

formule se réduit à

$$
\begin{gathered}
\text { (53.) } \quad H_{1}(0) \Theta(0) \Theta_{1}(0) \cdot \frac{H(u-a i)}{2 \Theta(a i) \Theta(u)} \\
=\frac{1}{i}\left[\frac{q^{\frac{1}{2}-\frac{1}{2} b} \cdot e^{i x}}{1-q^{1-b}}+\sum_{1}^{\infty} \frac{q^{m+\frac{1}{2}-\frac{1}{2} b} \cdot e^{(2 m+1) i x}}{1-q^{2 m+1-b}}-\sum_{1}^{\infty} \frac{q^{m-\frac{1}{2}+\frac{1}{2} b} \cdot e^{-(2 m-1) i x}}{1-q^{2 m-1+b}}\right] .
\end{gathered}
$$

En prenant la somme et la différence des formules (52. 53.), on obtiendra:

$$
\begin{gathered}
H_{1}(0) \Theta(0) \Theta_{1}(0) \cdot \frac{H(u+a i)+H(u-a i)}{2 \Theta(a i) \Theta(u)} \\
=\frac{2 q^{\frac{1}{2}-\frac{1}{2} b} \sin x}{1-q^{1-b}}+2 \sum_{1}^{\infty} \frac{q^{m+\frac{1}{2}-\frac{1}{2} b} \sin (2 m+1) x}{1-q^{2 m+1-b}}+2 \sum_{1}^{\infty} \frac{q^{m-\frac{1}{2}+\frac{1}{2} b} \sin (2 m-1) x}{1-q^{2 m-1+b}}, \\
H_{1}(0) \Theta(0) \Theta_{1}(0) \cdot \frac{H(u+a i)-H(u-a i)}{2 i \Theta(a i) \Theta(u)} \\
=\frac{2 q^{1-\frac{1}{2} b} \cos x}{1-q^{1-b}}+2 \sum_{1}^{\infty} \frac{q^{m+\frac{1}{2}-\frac{1}{2} b} \cos (2 m+1) x}{1-q^{2 m+1-b}}-2 \sum_{1}^{\infty} \frac{q^{m-\frac{1}{2}+\frac{1}{2} b} \cos (2 m-1) x}{1-q^{2 m-1+b}},
\end{gathered}
$$

et reunissant les termes analogues par rapport aux sin et cos, on aura enfin

$$
\begin{gathered}
H_{1}(0) \Theta(0) \Theta_{1}(0) \cdot \frac{H(u+a i)+H(u-a i)}{2 \Theta(a i) \Theta(u)} \\
=2\left(q^{\frac{1}{b} b}+q^{-\frac{1}{b} b}\right) \sum_{0}^{\infty} \frac{q^{\frac{1}{2}(2 m+1)}\left(1-q^{2 m+1}\right) \sin (2 m+1) x}{\left(1-q^{2 m+1+b}\right)\left(1-q^{2 m+1-b}\right)}, \\
H_{1}(0) \Theta(0) \Theta_{1}(0) \cdot \frac{H(u+a i)-H(u-a i)}{2 i \Theta(a i) \Theta(u)} \\
=2\left(q^{-\frac{1}{2} b}-q^{\frac{1}{b} b}\right) \sum_{0}^{\infty} \frac{q^{\frac{1}{2}(2 m+1)}\left(1+q^{2 m+1}\right) \cos (2 m+1) x}{\left(1-q^{2 m+1-b}\right)\left(1-q^{2 m+1+b}\right)} .
\end{gathered}
$$

Pour parvenir aux formules (7. et 8.) du No. cité des „Comptes rendus", il faut décomposer en fractions simples l'expression

$$
\frac{\Theta_{1}(u+a i)}{H_{1}(a i)}
$$

$=\frac{z^{\frac{1}{2}}(1+q v z)\left(1+q^{3} v z\right)\left(1+q^{5} v z\right) \ldots \times\left(1+q v^{-1} z^{-1}\right)\left(1+q^{3} v^{-1} z^{-1}\right)\left(1+q^{5} v^{-1} z^{-1}\right) \ldots}{q^{\frac{4}{4}}(1+z)\left(1+q^{2} z\right)\left(1+q^{4} z\right)\left(1+q^{6} z\right) \ldots \times\left(1+q^{2} z^{-1}\right)\left(1+q^{4} z^{-1}\right)\left(1+q^{6} z^{-1}\right) \ldots}$.

Posant

on trouve

$$
\frac{\Theta_{1}(u+a i)}{H_{1}(a i)}=\frac{z^{\frac{1}{2}}}{q^{\frac{z}{2}}}\left[\frac{A_{1}}{1+z}+\sum_{1}^{\infty} \frac{A_{m}}{1+q^{2 m} z}+\sum_{1}^{\infty} \frac{B_{m}}{z+q^{2 m}}\right]
$$

$$
\begin{gathered}
A_{0}=\frac{(1-q v)\left(1-q^{3} v\right)\left(1-q^{5} v\right) \ldots \times\left(1-q v^{-1}\right)\left(1-q^{3} v^{-1}\right)\left(1-q^{5} v^{-1}\right) \ldots}{\left(1-q^{2}\right)\left(1-q^{4}\right)\left(1-q^{6}\right) \ldots \times\left(1-q^{2}\right)\left(1-q^{4}\right)\left(1-q^{6}\right) \ldots} \\
=\frac{2 \Theta(u) q^{4}}{H_{1}(0) \Theta(0) \Theta_{1}(0)} \\
A_{m}=q^{m} v^{m} A_{0}, \quad B_{m}=q^{m} v^{-m} A_{0} ;
\end{gathered}
$$


10. Somoff, sur la théorie de la rotation d'un corps solide.

donc

$$
\begin{aligned}
& \text { (54.) } \quad H_{1}(0) \Theta(0) \Theta_{1}(0) \cdot \frac{\Theta_{1}(u+a i)}{2 H_{1}(a i) \Theta(u)} \\
= & \frac{z^{\frac{1}{2}}}{1+z}+\sum_{1}^{\infty} \frac{q^{m} v^{m} z^{\frac{1}{2}}}{1+q^{2 m} z}+\sum_{1}^{\infty} \frac{q^{m} v^{-m} z^{-\frac{1}{2}}}{1+q^{2 m} z^{-1}} \\
= & \frac{q^{\frac{1}{2} b}}{1+q^{b}}+\sum_{1}^{\infty} \frac{q^{m+\frac{1}{2} b} \cdot e^{2 m i x}}{1+q^{2 m+b}}+\sum_{1}^{\infty} \frac{q^{m-\frac{1}{2} b} \cdot e^{-2 m i x}}{1+q^{2 m-b}}
\end{aligned}
$$

d'où l'on tire, en changeant $\boldsymbol{u}$ en $-\boldsymbol{u}$,

$$
\begin{aligned}
\text { (55.) } \quad H_{1}(0) \Theta(0) \Theta_{1}(0) \cdot \frac{\Theta_{1}(u-a i)}{2 H_{1}(a i) \Theta(u)} \\
=\frac{q^{\frac{1}{2} b}}{1+q^{b}}+\sum_{1}^{\infty} \frac{q^{m+\frac{1}{2} b} \cdot e^{-2 m i x}}{1+q^{2 m+b}}+\sum_{1}^{\infty} \frac{q^{m-\frac{1}{2} b} \cdot e^{2 m i x}}{1+q^{2 m-b}} .
\end{aligned}
$$

En vertu de ces deux formules on a

$$
\begin{aligned}
& H_{1}(0) \Theta(0) \Theta_{1}(0) \cdot \frac{\Theta_{1}(u+a i)+\Theta_{1}(u-a i)}{2 H_{1}(a i) \Theta(u)} \\
= & \frac{2 q^{\frac{1}{2} b}}{1+q^{b}}+2\left(q^{\frac{1}{2} b}+q^{-\frac{1}{2} b}\right) \sum_{1}^{\infty} \frac{q^{m}\left(1+q^{2 m}\right) \cos (2 m x)}{\left(1+q^{2 m+b}\right)\left(1+q^{2 m-b}\right)} \\
& H_{1}(0) \Theta(0) \Theta_{1}(0) \cdot \frac{\Theta_{1}(u-a i)-\Theta_{1}(u+a i)}{2 i H_{1}(a i) \Theta(u)} \\
= & \left(q^{-\frac{1}{2} b}-q^{\frac{1}{2} b}\right) \sum_{1}^{\infty} \frac{q^{m}\left(1-q^{2 m}\right) \sin (2 m x)}{\left(1+q^{2 m+b}\right)\left(1+q^{2 m-b}\right)}
\end{aligned}
$$

Au moyen de la formule (55.), changeant $a$ en $K^{\prime}-a$ et $b$ en 1 -b, on obtient

(56). $H_{1}(0) \Theta(0) \Theta_{1}(0) \cdot \frac{H_{1}(u+a i)}{2 \Theta_{1}(a i) \Theta(u)}=H_{1}(0) \Theta(0) \Theta_{1}(0) \cdot \frac{e^{-i x} \Theta_{1}\left[u-i\left(K^{\prime}-a\right)\right]}{H_{1}\left[i\left(K^{\prime}-a\right)\right] \Theta(u)}$

$$
=\frac{q^{\frac{1}{2}-\frac{1}{2} b} \cdot e^{-i x}}{1+q^{1-b}}+\sum_{1}^{\infty} \frac{q^{m+\frac{1}{2}-\frac{1}{2} b} \cdot e^{-(2 m+1) i x}}{1+q^{2 m+1-b}}+\sum_{1}^{\infty} \frac{q^{m-\frac{1}{2}+\frac{1}{2} b} \cdot e^{(2 m-1) i x}}{1+q^{m-1+b}},
$$

et, changent $u$ en $-u$ dans cette dernière formule, on en déduit

$$
\begin{gathered}
\text { (57.) } \quad H_{1}(0) \Theta(0) \Theta_{1}(0) \cdot \frac{H_{1}(u-a i)}{2 \Theta_{1}(a i) \Theta(u)} \\
=\frac{q^{1-\frac{1}{2} b} \cdot e^{i x}}{1+q^{1-b}}+\sum_{1}^{\infty} \frac{q^{m+\frac{1}{2}-\frac{1}{2} b} \cdot e^{(2 m+1) i x}}{1+q^{2 m+1}-b}+\sum_{1}^{\infty} \frac{q^{m-\frac{1}{2}+\frac{1}{2} b} \cdot e^{-(2 m-1) x}}{1+q^{2 m-1+b}} .
\end{gathered}
$$

Prenant la somme et la différence des valeurs (56. 57.) et réunissant les termes analogues par rapport aux sin et cos, on trouve enfin 
10. Somoff, sur la théorie de la rotation d'un corps solide.

$$
\begin{gathered}
H_{1}(0) \Theta(0) \Theta_{1}(0) \cdot \frac{H_{1}(u+a i)+H_{1}(u-a i)}{2 \Theta_{1}(a i) \Theta(u)} \\
=2\left(q^{-\frac{1}{2} b}+q^{\frac{1}{2} b}\right) \sum_{0}^{\infty} \frac{q^{\frac{1}{2}(2 m+1)}\left(1+q^{2 m+1}\right) \cos (2 m+1) x}{\left(1+q^{2 m+1-b}\right)\left(1+q^{2 m-1+b}\right)} \\
H_{1}(0) \Theta(0) \Theta_{1}(0) \cdot \frac{H_{1}(u+a i)-H(u-a i)}{2 i \Theta_{1}(a i) \Theta(u)} \\
=2\left(q^{-\frac{1}{2} b}-q^{\frac{1}{2} b}\right) \sum_{0}^{\infty} \frac{q^{\frac{1}{2}(2 m+1)}\left(1-q^{2 m+1}\right) \sin (2 m+1) x}{\left(1+q^{2 m+1-b}\right)\left(1+q^{2 m+1+b}\right)} .
\end{gathered}
$$

Il est facile de développer aussi en série assez convergeante la valeur de $\boldsymbol{n}^{\prime}$, qui détermine la rotation de l'axe $\boldsymbol{O} x$ dans le plan invariable. Pour cela développons les valeurs

$$
\frac{\partial \log H(a i)}{\partial a}, \quad \frac{\partial \log \Theta(a i)}{\partial a} \text {. }
$$

Or

$$
\frac{\partial \log \Theta(u)}{\partial u}=\frac{\partial\left(\log \frac{\Theta(u)}{\Theta(0)}\right)}{\partial u}=\mathbf{Z}(\boldsymbol{u}),
$$

et par la formule (1.) du \$.47. des "Fundam. nova" on a

$$
\mathbf{Z}(u)=\frac{2 \pi}{K}\left[\frac{q \sin 2 x}{1-q^{2}}+\frac{q^{2} \sin 4 x}{1-q^{4}}+\frac{q^{3} \sin 6 x}{1-q^{6}}+\cdots\right]
$$

posant $u=a i$, on trouvera

$$
\frac{\partial \log \Theta(a i)}{\partial a}=\frac{\pi}{K}\left[\frac{q\left(q^{b}-q^{-b}\right)}{1-q^{2}}+\frac{q^{2}\left(q^{2 b}-q^{-2 b}\right)}{1-q^{4}}+\frac{q^{3}\left(q^{3 b}-q^{-3 b}\right)}{1-q^{6}}+\cdots\right]
$$

Par une des formules (page 101) du No. cité des „Comptes rendus", on a

$$
H(\boldsymbol{a i})=i g \Theta\left[i\left(\boldsymbol{K}^{\prime}-a\right)\right], \quad \text { où } g=e^{-\frac{\pi K^{\prime}}{4 K}+\frac{a \pi}{2 K}}=q^{\frac{a}{2 \pi}} e^{\frac{a \pi}{2 K}}
$$

par suite

$$
\begin{aligned}
& \log H(a i)=\log \left(i q^{\frac{4}{4}}\right)+\frac{\pi a}{2 K}+\log \Theta\left[i\left(K^{\prime}-a\right)\right] \\
& \frac{\partial \log H(a i)}{\partial a}=\frac{\pi}{2 K}+\frac{\partial \log \Theta\left[i\left(K^{\prime}-a\right)\right]}{\partial a}=\frac{\pi}{2 K}-\frac{\partial \log \Theta\left[i\left(K^{\prime}-a\right)\right]}{\partial\left(K^{\prime}-a\right)} .
\end{aligned}
$$

Pour avoir le développement du dernier terme de cette formule, on n'a qu'à changer $b$ en $1-b$ dans la formule (58.). Cela donne

$$
\begin{gathered}
\frac{\partial \log H(a i)}{\partial a}=\frac{\pi}{K}\left[\frac{1}{2}-\frac{q\left(q^{1-b}-q^{-1+b}\right)}{1-q^{2}}-\frac{q^{2}\left(q^{2-2 b}-q^{-2+2 b}\right)}{1-q^{4}}-\cdots\right] \\
=\frac{\pi}{K}\left[\frac{1}{2}+\frac{q^{b}\left(1-q^{2-2 b}\right)}{1-q^{2}}+\frac{q^{2 b}\left(1-q^{4-4 b}\right)}{1-q^{4}}+\cdots\right]
\end{gathered}
$$


10. Somoff, sur la théorie de la rotation d'un corps solide.

Donc

$$
\begin{gathered}
n^{\prime}=\frac{1}{A-C}\left[C \frac{\partial \log H(a i)}{\partial a}-A \frac{\partial \log \Theta(a i)}{\partial a}\right] \\
=\frac{1}{A-C} \cdot \frac{\pi}{K}\left[\frac{1}{2} C+\frac{C q^{b}\left(1-q^{2-2 b}\right)+A q^{1-b}\left(1-q^{2 b}\right)}{1-q^{2}}+\cdots\right]
\end{gathered}
$$

Observons que cette valeur est toujours positive: donc le mouvement de $\boldsymbol{O} x$ se fait toujours dans le même sens.

Développons encore la valeur angulaire $\Omega$, qui sert à déterminer la position de la ligne mobile des noeuds par rapport à l'axe $O x$. On a trouvé plus haut

$$
\Omega=\frac{1}{2} i \log \frac{\Theta(u-a i)}{\Theta(u+a i)},
$$

et d'après les formules du $\$$.52. des "Fundam. nova" on a $\log \frac{\Theta(u)}{\Theta(0)}=\int_{0}^{u} \boldsymbol{Z}(u) \partial u=-2\left[\frac{q \cos 2 x}{1-q^{2}}+\frac{q^{2} \cos 4 x}{2\left(1-q^{4}\right)}+\frac{q^{3} \cos 6 x}{3\left(1-q^{6}\right)}+\cdots \cdot\right]+$ const. De là, en substituant $u \mp a i$ au lieu de $u$, on obtient $\log \Theta(u-a i)=-\left[\frac{q\left(e^{2 i x} \cdot q^{-b}+e^{-2 i x} \cdot q^{b}\right)}{1-q^{2}}+\frac{q^{2}\left(e^{4 i x} \cdot q^{-2 b}+e^{-4 i x} \cdot q^{2 b}\right)}{2\left(1-q^{4}\right)}+\cdots\right]+$ const. $\log \Theta(u+a \dot{i})=-\left[\frac{q\left(e^{-2 i x} \cdot q^{-b}+e^{2 i x} \cdot q^{b}\right)}{1-q^{2}}+\frac{q^{2}\left(e^{-4 i x} \cdot q^{-2 b}+e^{4 i x} \cdot q^{2 b}\right)}{2\left(1-q^{4}\right)}+\cdots\right]+$ const. par conséquent

$$
\Omega=\frac{1}{2} i \log \frac{\Theta(u-a i)}{\Theta(u+a i)}=\frac{q^{1-b}\left(1-q^{2 b}\right)}{1-q^{2}} \sin 2 x+\frac{q^{2-2 b}\left(1-q^{4 b}\right)}{2\left(1-q^{4}\right)} \sin 4 x+\cdots
$$

Cette valeur peut aussi être exprimé par une fonction elliptique de troisième espèce, savoir

(59.) $\Omega=\frac{1}{2} i \log \frac{\Theta(u-a i)}{\Theta(u+a i)}=i \Pi(u, a i)-i u \mathbf{Z}(a i)$ (Fundam. nova p. 146) où

$$
\begin{aligned}
\Pi(u, a i) & =\int_{0}^{u} \frac{k^{2} \sin \mathrm{am}(a i) \cos \mathrm{am}(a i) \Delta \mathrm{am}(a i) \sin ^{2} \mathrm{am}(u) \partial u}{1-k^{2} \sin ^{2} \mathrm{am}(a i) \sin ^{2} \mathrm{am}(u)}, \\
\boldsymbol{Z}(\boldsymbol{a i}) & =\frac{1}{K} \int_{0}^{K} \frac{k^{2} \sin \mathrm{am}(a i) \cos \mathrm{am}(a i) \Delta \mathrm{am}(a i) \sin ^{2} \mathrm{am}(u) \partial u}{1-k^{2} \sin ^{2} \mathrm{am}(a i) \sin ^{2} \mathrm{am}(u)} .
\end{aligned}
$$

La valeur de $\Omega$ est nulle d'abord à l'instant $t=0$; parcequ'alors $\boldsymbol{u}=0, x=0$. Puis elle croît et atteint un maximum, pour lequel la dérivée de (59.) est nulle. Donc, pour déterminer la valeur de $u$, qui repond 
à ce maximum, on aura

$$
\frac{i k^{2} \sin \operatorname{am}(a i) \cos \mathrm{am}(a i) \Delta \operatorname{am}(a i) \sin ^{2} \mathrm{am}(u)}{1-k^{2} \sin ^{2} \mathrm{am}(a i) \sin ^{2} \mathrm{am}(u)}=i \mathbf{Z}(a i)=\frac{\partial \log \Theta(a i)}{\partial a},
$$

ou bien

$$
\frac{k^{2} \sin ^{2} \operatorname{am}(a i) \sin ^{2} \operatorname{am}(u)}{1-k^{2} \sin ^{2} a m(a i) \sin ^{2} \operatorname{am}(u)}=\frac{\operatorname{tg} \operatorname{am}(a i)}{i \Delta \operatorname{am}(a i)} \frac{\partial \log \Theta(a i)}{\partial a}=\frac{\frac{\partial \log \Theta(a i)}{\partial a}}{\frac{\partial \log H(a i)}{\partial a}-\frac{\partial \log \Theta(a i)}{\partial a}},
$$

d'où l'on tire

$$
\sin \operatorname{am}(\boldsymbol{u})= \pm \frac{1}{k \sin \operatorname{am}(a i)} \cdot \sqrt{\frac{\frac{\partial \log \Theta(a i)}{\partial a}}{\frac{\partial \log H(a i)}{\partial a}}}= \pm \frac{\Theta(a i)}{\sqrt{k \cdot H(a i)}} \cdot \sqrt{\frac{\frac{\partial \log \Theta(a i)}{\partial a}}{\frac{\partial \log H(a i)}{\partial a}}}
$$

Par cette formule on trouve $u$, et par suite $t=\frac{u}{n}$, qui repondent au maximum de $\Omega$.

A l'instant $t=\frac{K}{n}$ la valeur de $\Omega$ s'évanouit encore, parcequ'alors $\Omega=\frac{1}{2} i \log \frac{\Theta(K-a i)}{\Theta(K+a i)}=i \Pi(K, a i)-i K \mathbf{Z}(a i)=i K \mathbf{Z}(a i)-i K \mathbf{Z}(a i)=0 ;$ après quoi elle réprend les mêmes valeurs dans le sens négatif; et ainsi de suite.

Il est facile de discuter aussi les autres circonstances du mouvement. St. Petersbourg le 20 Mai 1850. 